\title{
Properties of highly electronegative plasmas produced in a multipolar magnetic-confined device with transversal magnetic filter
}

\author{
Mihai Draghici ${ }^{\text {a) }}$ and Eugen Stamate ${ }^{\text {b) }}$ \\ Plasma Physics and Technology Programme, Ris $\varnothing$ National Laboratory for \\ Sustainable Energy, Technical University of Denmark, Frederiksborgvej 399, \\ DK-4000 Roskilde, Denmark
}

\begin{abstract}
Highly electronegative plasmas were produced in $\mathrm{Ar} / \mathrm{SF}_{6}$ gas mixtures in a DC discharge with multipolar magnetic confinement and transversal magnetic filter. Langmuir probe and mass spectroscopy were used for plasma diagnostics. Plasma potential drift, the influence of small or large area biased electrodes on plasma parameters, the formation of the negative ion sheath, and etching rates by positive and negative ions have been investigated for different experimental conditions. Reducing the electron temperature below $1 \mathrm{eV}$ the density ratio of negative ion to electron exceeded 100 even for very low amounts of $\mathrm{SF}_{6}$ gas. The plasma potential drift could be controlled by proper wall conditioning. A large-electrode biased positively had no effect on plasma potential for density ratios of negative ion to electrons larger than 50. For similar electronegativities or higher a negative ion sheath could be formed by applying a positive bias of a few hundred volts.
\end{abstract}

Keywords: negative ions, ion-ion plasma, $\mathrm{Ar} / \mathrm{SF}_{6}$ plasma, plasma diagnostics

PACS:

Permanent address: National Institute of Materials Physics, P.O. box MG-7, Bucharest-Magurele, 077125, Romania
E-mail corresponding author: eust@ risoe.dtu.dk 


\section{Introduction}

Electronegative plasmas produced by attaching mobile low-energy electrons to create heavy negative ions are very attractive for both fundamental research and plasma processing applications [1,2]. Recent reports include the discrete and modal focusing effects for negative ions in three-dimensional plasma sheath lenses [3,4] that can be used for negative ion beam extraction [5], and mass spectrometry [6]. Highly electronegative discharges, also called ion-ion plasmas [7-9], are seen as a promising solution for electric propulsion [10-13], and neutral beam development [14]. Positive ion sheath formed when applying a negative bias with respect to plasma potential, $V_{\mathrm{p}}$, has been extensively investigated starting with the pioneering work of Langmuir. Recently, there is also a growing interest in electron sheath [15] and its transition to anodic glow [16-18]. However, there is very limited understanding of the negative charge sheath formed by both electron and negative ion. Electronegative plasmas produced in argon and sulphur hexafluoride $\left(\mathrm{SF}_{6}\right)$ are used to etch silicon [19], silicon nitride [20], silicon carbide [21-24] and titanium carbide [25] with high etch rates and without film residues. Nevertheless, problems associated with charge accumulation at the trench bottom during high-aspect-ratio etching by positive ions have led a systematic research on silicon etching by negative ions [26-29]. The goal was to use the benefit of a considerably lower charging potential during negative ion neutralization on the substrate. Negative ion etching with low energies and low charging potentials is also attractive for low damage processing required in node technologies below $50 \mathrm{~nm}$ [30]. So far, despite of good theoretical coverage [31-35] and a large variety of methods for negative ion diagnostic including optical emission [36], photodetachment [37], Langmuir probe [38-43], thermal probe [44], propagation of the ion acoustic waves [45-47] and Thomson scattering [48] there are still important basic questions related to highly electronegative discharges that need to be answered.

The aim of this paper is to clarify some physical aspects related to highly electronegative discharges, including plasma potential drift, the influence of small or large area biased electrodes on plasma parameters, the formation of the negative ion sheath, and etching rates and surface roughness by positive and negative ion impact. Experiments are performed in $\mathrm{Ar} / \mathrm{SF}_{6}$ plasma produced in a multipolar magnetically confined discharge with transversal magnetic filter.

\section{Experimental}

The reactor chamber has a cylindrical geometry and it is schematically illustrated in figure 1(a). The experiments were performed using $\mathrm{Ar} / \mathrm{SF}_{6}$ gases and the DC plasma was produced between a negatively biased filament, and a grounded anode. An inner metallic cylinder, $\mathrm{C}_{1}$, could be polarized at a certain 
potential $U_{\mathrm{W}}$ and it was used to study the possibility of tuning the plasma potential. Moreover, $\mathrm{C}_{1}$ could be heated up to a few hundred degrees using a vacuum compatible heating system sandwiched between $\mathrm{C}_{1}$ and an additional cylinder, $\mathrm{C}_{2}$, electrically insulated from the wall. The pressure inside the main chamber during the measurements was 3 mTorr and the discharge voltage $150 \mathrm{~V}$. A transversal magnetic filter divides the chamber into two regions: source plasma (SP) and target plasma (TP) [49-51]. It was made of permanent magnets (2000 Gauss at the surface) placed in rectangular pipes situated $45 \mathrm{~mm}$ apart. Cooling water was flowing through the filter during plasma operation to avoid damage of the magnets by excessive heating. The magnetic field between two consecutive pipes was about 500 Gauss. The magnetic filter reduces the electron temperature, $T_{\mathrm{e}}$, by increasing the diffusion time of the electrons in SP. Consequently, the negative ion production becomes much more efficient in TP. The magnetic confinement at the walls is obtained with an array of permanent magnets which are fixed at the outer side of the cylinder.

A mass spectrometer Hiden EPIC Series 1000 was used to record and monitor both positive and negative ion species. The plasma parameters were measured using two Langmuir cylindrical probes: one situated in SP, which has a fixed position and the other in TP, which can be moved in radial direction. The electrical probe circuit and the details of the "Test Function" procedure for extracting the bulk and hot electron densities $\left(n_{\mathrm{e}}\right.$ and $\left.n_{\mathrm{eh}}\right)$ and temperatures $\left(T_{\mathrm{e}}\right.$ and $\left.T_{\mathrm{eh}}\right)$ and the negative ion parameters $\left(n_{\mathrm{ni}}\right.$ and $\left.T_{\mathrm{ni}}\right)$ are presented in detail elsewhere [39, 40]. Special care was devoted to avoid recording erroneous probe characteristics affected by contaminations effects reported for reactive plasmas [52, 53]. It has been shown that the DC discharges with magnetic confinement exhibit three groups of electrons, namely the anisotropic primary electrons emitted by filament and accelerated to an energy correlated to the discharge voltage in a very thin sheath surrounding the cathode (filament) [54]. The isotropic hot electrons resulted from primary electrons after repetitive scattering in the magnetic confinement near the walls and consecutive ionization with the buffer gas and the bulk, cold isotropic electrons, resulted by ionization. For simplicity, this paper presents the total electron density as the sum of hot and bulk density while the effective temperature, $T_{\text {eff }}$, obtained by integrating the electron energy distribution function, $T_{\text {eff }}=2 / 3\langle\angle\rangle$, is used as a common parameter for electron temperature where $\langle\zeta\rangle$ is the average electron energy. To prevent the primary electrons from reaching the TP and suppressing instabilities occurring in the electronegative regime, a stainless steel mesh with $1 \times 1 \mathrm{~mm}$ grid size was placed between the filament and the transversal magnetic filter. The mesh also increases considerably the life time of the filament by reducing its etching.

\section{Results and discussion}

Figure 2 presents (a) the ratio of the negative ions to electrons $\_n_{\mathrm{ni}} /\left(n_{\mathrm{e}}+n_{\mathrm{eh}}\right)$, (b) the positive ion density and (c) the effective electron temperature as function of $\mathrm{SF}_{6}$ flow rate for two different discharge currents, $150 \mathrm{~mA}$ and $500 \mathrm{~mA}$ in both SP and TP regions for a constant Ar flow of $2 \mathrm{sccm}$. Higher $T_{\text {eff }}$ and $n_{\mathrm{i}}$ values in SP result in a less efficient negative ion production, while $T_{\text {eff }}$ lower than $1 \mathrm{eV}$ in TP leads to $\square$ 
larger than 100 for $\mathrm{SF}_{6}$ flows over $0.2 \mathrm{sccm}$. This fact is well correlated with the cross section of $\mathrm{F}^{\square}, \mathrm{SF}_{5}^{\square}$ and $\mathrm{SF}_{6}^{\square}$, which are very large for $T_{\text {eff }}<1 \mathrm{eV}$ [55]. For $150 \mathrm{~mA}$ and a $\mathrm{SF}_{6}$ flow of $0.5 \mathrm{sccm}$, we could obtain a negative ion to electron density ratio of 300 in a continuous operation regime without filtering out the electrons. This proves that this type of discharges with a transversal magnetic filter is very efficient in producing almost electron free plasmas (ion-ion plasmas) at densities below $10^{16} \mathrm{~m}^{-3}$. Figure 3 illustrates the mass spectrometry results for the negative ion species produced in TP, namely the dependence of the measured peaks of $\mathrm{F}^{\square}, \mathrm{SF}_{5}^{\square}$ and $\mathrm{SF}_{6}^{\square}$ as a function of the flow rate of $\mathrm{SF}_{6}$ for $150 \mathrm{~mA}$ and $500 \mathrm{~mA}$ discharge current. When the discharge current is increased, the maxima shift toward larger $\mathrm{SF}_{6}$ flow rates and they become higher for higher discharge currents. The most efficiently produced specie is $\mathrm{F}^{\square}$, while $\mathrm{SF}_{6}^{\square}$ has the lowest efficiency.

The plasma potential for different flow rates of $\mathrm{Ar}$ and $\mathrm{SF}_{6}$ while keeping the flow ratio constant at 8 is presented in figure 4 (a). Due to fast adsorption at the walls and also possible deposition of various species of ions and radicals produced in $\mathrm{Ar} / \mathrm{SF}_{6}$ plasma, one finds a drift of $V_{\mathrm{pl}}$ toward negative values, which depends on the flow rate of $\mathrm{SF}_{6}$. Keeping the discharge "on" further lowers $V_{\mathrm{pl}}$ to values that reduce the effective applied voltage with respect to ground (anode) below the threshold of about $40 \mathrm{~V}$ necessary to sustain the plasma. The time dependence of $\square$ parameter corresponding to data shown in figure 4 (a) is presented in figure 4 (b). After running continuously the plasma only in Ar gas for a few hours the plasma potential is reversibly getting back to the initial starting value and the next curve corresponding to different $\mathrm{Ar}$ and $\mathrm{SF}_{6}$ flow rates was measured. One can see that a high value of a few hundreds for the ratio of the negative ions to electrons is obtained only for $\mathrm{SF}_{6}$ flow rates larger than of $0.125 \mathrm{sccm}$. This suggests the role of an accumulation process that can take place at the walls but also in the volume as the heavy $\mathrm{SF}_{6}$ molecules are evacuated at lower rates than Ar. The rather constant values of $\square$ in time shows that the plasma potential drift has no major influence on the production mechanism of negative ions. However, the drift of $V_{\mathrm{pl}}$ spoils the benefit of very large $\square$ available in a continuous mode of operation, not pulsed as in ion-ion plasma produced in afterglow discharges, which is desired in many applications and basic studies. To verify the influence of the walls state over the potential drift, we performed a very good cleaning process of the chamber and repeated the measurements. In figure 4 (a) the plasma potential drift after 30 min is larger than $30 \mathrm{~V}$ for $0.25 \mathrm{sccm} \mathrm{SF}_{6}$ flow rate. According to figure 4 (c) after cleaning the chamber, the plasma potential remains at the constant value of $0.32 \mathrm{~V}$ in the first $30 \mathrm{~min}$ and decreases to only $-1.2 \mathrm{~V}$ after $120 \mathrm{~min}$, in spite of the fact that the $\mathrm{SF}_{6}$ flow rate is 4 times higher. To confirm that the change in sign of $V_{\mathrm{pl}}$ does not result in significant changes of plasma parameters the plasma density was also included in figure 4 (c). The density was slightly increasing in time due to walls conditioning by plasma heating after switching on the discharge. 
The mesh placed in front of the cathode had an essential role in extending the lifetime of the filament from about 10 minutes to several hours and also to improve the discharge stability. In spite of this advantage, the mesh was also affected by a faster contamination taking place at high temperatures by discharge species including sulphur.

In order to clarify the role of physical adsorption at the walls, plasma potential relaxation was also studied, and this process is illustrated in figure 5. For these measurements, $\mathrm{Ar}$ and $\mathrm{SF}_{6}$ were introduced into the chamber and the plasma was produced until the plasma potential became $-20 \mathrm{~V}$. Then the $\mathrm{SF}_{6} \mathrm{was}$ stopped and the measurements were performed only in Ar plasma. Heating the metallic inner cylinders $\mathrm{C}_{1}$ and $\mathrm{C}_{2}$ to $150^{\circ} \mathrm{C}$, one can see that some species adsorbed on the walls are released and the plasma potential goes back faster toward the initial positive value, showing that the adsorption is also responsible for the plasma drift.

Plasma sources are often used for positive ion beam extraction where the ion energy can be adjusted by controlling the potential difference between $V_{\mathrm{pl}}$ and the extraction bias. In our experiment one possibility of such control is to bias the inner metallic cylinder, $C_{1}$, so as the plasma potential to follow the potential of the most positive electrode or section of the wall [56]. This possibility is valid for electropositive plasmas, which contain very mobile negative charges (electrons) with respect to positive ions, but it needs to be tested for electronegative discharges. For this reason, we studied the dependence of $V_{\mathrm{pl}}$ as a function of the bias applied to the inner cylinder $U_{\mathrm{W}}$ for different $\mathrm{SF}_{6}$ flows and it is shown in figure 6 (a) where the values in branches correspond to $\square$ An interesting fact is the presence of a jump at a certain $U_{\mathrm{W}}$ bias, which is mainly determined by the $\mathrm{SF}_{6}$ flow rate or $\square$ For small biases applied to the cylinder, the plasma potential is almost constant. Then, depending on the $\mathrm{SF}_{6}$ flow rate, $V_{\mathrm{pl}}$ jumps and becomes approximately equal to $U_{\mathrm{W}}$ bias as one would expect and the increases linearly with $U_{\mathrm{W}}$. When the $\mathrm{SF}_{6}$ flow rate is increased to 0.1 sccm ( $\left.\_60\right)$ no jump is noticed for $U_{\mathrm{W}}$ biases less than $50 \mathrm{~V}$. The corresponding ion densities are illustrated in figure 6 (b) and show a large increase for lower $\mathrm{SF}_{6}$ flows. It is evident that the fast electron and the ion flux change when biasing $\mathrm{C}_{1}$ and this change can also be discontinuous. For example, a sharp increase in the near-wall potential associated with self trapping of fast electrons in plasma volume was reported by Demidov et al. [57]. The jump can also be related to a change in the current closure to the anode as reported by Dorf et al. in [15]. Experiments with conducting walls performed by Stangeby and Allen [58] revealed the current amplifier effect when changing the wall potential, a situation that is different from the present one where the target plasma has a diameter larger than its length. Thus, further investigations are necessary in order to correlate the jump in figure 6 with a detailed phenomenology. We checked $T_{\text {eff }}$ before and after the jump and the radial profile measurements for Ar plasma are presented in figure 7. There are no large differences between the curves corresponding to before $\left(U_{\mathrm{W}}=10 \mathrm{~V}\right)$ and after the jump $\left(U_{\mathrm{W}}=25 \mathrm{~V}\right)$, proving that the jump does not affect the electron temperature. The radial profile measurements of the plasma potential show also that this parameter is constant. These results demonstrate that one cannot control the plasma potential in a highly electronegative discharge by 
biasing positively a large electrode exposed to plasma. This limits the possibility to control the energy of negative ions extracted from plasma using $V_{\mathrm{p}}$, but favours the applicability of these plasmas for negative ion etching, where a large surface (the wafer) needs to be biased positively as to provide the anisotropic flux of negative ions necessary for directive etching without affecting the plasma potential.

It was recently demonstrated that a negatively biased electrode interfacing an insulator, for example, one side conductive electrode, or a conducting surface surrounded by an insulator, creates in plasma a potential structure that in certain conditions exhibits discrete and modal focusing effects [3, 4]. If the applied bias is positive with respect to plasma potential then the same phenomenology affects the negative ions. Based on it, some new applications have been developed related to negative ion diagnostics [59], negative ion extraction [5] and mass spectrometry [6]). Since these applications are using negative sheath formed at positively biased electrodes of sizes significantly lower than the plasma volume, it become important to know in what conditions one can create such sheath in electronegative plasmas and how these structures influences the plasma parameters. The $I-V$ characteristics measured in Ar plasma only for $V \sqsubset 0$ $\left(V_{\mathrm{pl}}=\square 1.5 \mathrm{~V}\right.$ ) using a planar probe (PP) of $10 \mathrm{~mm}$ in diameter mounted in the TP are illustrated in figure 8. The family of $I-V$ characteristics recorded with PP exhibits different regions. After a fast increase of the current with the applied voltage up to $10 \mathrm{~V}$ and a saturation-like region where the current increases slowly and almost linear with the voltage, the current jumps suddenly to high values corresponding to the so-called glow discharge or anodic-glow [16-18, 60]. The voltage applied to the probe, for which the glow discharge appears, decreases with the increase of the plasma density (larger discharge currents) as expected from Townsend theory. Due to this behaviour it is difficult to create thick negative sheath of electrons at pressures from 1 to 100 mTorr, a range that is very interesting both for applications and basic research. By replacing the electrons with heavy negative ions, one expects a clear change in the negative sheath structure as well as in the formation of the anodic-glow. For example, figure 9 shows the $I-V$ characteristics in $\operatorname{Ar} / \mathrm{SF}_{6}$ plasma $\left(V_{\mathrm{pl}}=\square 1.5 \mathrm{~V}\right)$ measured on the planar probe for different $\mathrm{SF}_{6}$ flow rates and Ar flow of 2 sccm for a discharge current of $500 \mathrm{~mA}$. The density ratio of negative ions to electrons, $\square$ is included in parenthesis for each curve. Thus, the ignition bias for the anodic glow shifts from $80 \mathrm{~V}$ for $\_9$ to $320 \mathrm{~V}$ for $\exists 34$ and there is now glow ignition for $\triangle>37$ up to $400 \mathrm{~V}$. This fact is directly correlated with a decrease in electron density resulted from negative ion production and proves that one can create a thick negative sheath dominated by negative ions for $\triangle 50$. Moreover, it suggests that it should be possible to implement a model for correlating the ignition bias of the anodic-glow with $\square$ as a method for negative ion diagnostics in low electronegative discharges. As shown in figure 6 a large electrode biased positively affects plasma parameters for $\angle<50$, but it is expected that a smaller electrode to have a limited influence. To verify this hypothesis we have measured $V_{\mathrm{pl}}$ and $\square$ as a function of $\mathrm{SF}_{6}$ flow for $2 \mathrm{sccm}$ of Ar by placing the cylindrical probe LP1 at $20 \mathrm{~mm}$ from the planar electrode when PP was grounded $(0 \mathrm{~V})$ or biased at $200 \mathrm{~V}$. The $200 \mathrm{~V}$ bias was applied only for $\mathrm{SF}_{6}$ flows that inhibited the glow ignition, respectively $₫ 50$ or $\mathrm{SF}_{6}$ flows larger 
than $0.1 \mathrm{sccm}$. The result is shown in figure 10 and demonstrates that one can form a negative sheath to a small electrode with respect to chambers walls with negligible changes of the plasma parameters. Because of the drift of the plasma potential in time, these measurements were performed starting in Ar plasma with the same value of the plasma potential. Then, every point was measured after the same interval of time.

At plasma densities below $10^{16} \mathrm{~m}^{-3}$ one expects low etching rates for both positive and negative ions. Etching rates by negative ions have been previously reported in ICP [26] or after glow discharges [8]. However, since the current setup was improved to provide a negligible $V_{\mathrm{pl}}$ drift during 1 hour of operation for $\triangle 100$ we could also confirm the etching rates and the resulted surface morphology. The etching rates on silicon corresponding to both positive and negative ions for two values of the discharge current (150 and $500 \mathrm{~mA}$ ) as a function of ion energy are presented in figure 11, where $\mathrm{Ar}=2 \mathrm{sccm}, \mathrm{SF}_{6}=0.5 \mathrm{sccm}, \quad \neq 206$ for $I_{\mathrm{d}}=500 \mathrm{~mA}$ and $Z 301$ for $I_{\mathrm{d}}=150 \mathrm{~mA}$. For all measured points, the etching rate by negative ions was slightly below of than that by positive ions with a value of $45 \mathrm{~nm} / \mathrm{min}$ measured for $500 \mathrm{~mA}$ and $250 \mathrm{eV}$. Etching with $13 \mathrm{~nm} / \mathrm{min}$ was also observed for $500 \mathrm{~mA}$ and $0 \mathrm{eV}$ (the sample was kept at plasma potential) by plasma radicals. A high electron fraction present for low $\square$ can bring a large thermal flux when biasing the substrate at $250 \mathrm{~V}$. However, it has been shown that for $\triangle>100$ the heat flux is reduced to values comparable to those resulted by positive ion [44]. For example, monitoring the substrate temperature at 500 $\mathrm{mA}$ and $250 \mathrm{eV}$ we could see an increase of only $10 \llbracket \mathrm{C}$ during negative ion etching comparative with positive ion etching. Not only low charging, but also less damage is targeted by negative ion etching. Thus, it is important to check on the resulted surface morphology by the two types of ions. The AFM measurements presented in figure 12 for $\mathrm{Ar}=2 \mathrm{sccm}, \mathrm{SF}_{6}=0.5 \mathrm{sccm}, 500 \mathrm{~mA}$ discharge current, $50 \mathrm{eV}$ and $\exists 206$ show that the negative ion etching resulted in a surface characterized by a root mean square (RMS) of $12.6 \mathrm{~nm}$ while for positive ions the RMS was $34.9 \mathrm{~nm}$.

\section{Summary and conclusions}

Highly electronegative plasmas with a density ratio of the negative ion to electron larger than a few hundreds were investigated in $\mathrm{Ar} / \mathrm{SF}_{6}$ gas mixtures in DC plasma with multipolar magnetic confinement and transversal magnetic filter. The main negative ion species were $\mathrm{F}^{\square}, \mathrm{SF}_{5}^{\square}$ and $\mathrm{SF}_{6}^{\square}$. The drift of the plasma potential in time was compensated with proper wall cleaning and temperature enhanced gas desorption. The effect of large or small electrodes biased positively with respect to $V_{\mathrm{pl}}$ on electronegative plasma parameters was investigated and the results prove the possibility to create centimetre-size sheaths dominated by negative ions without igniting anodic glows, elevating plasma potential or bringing high thermal fluxes to the substrate as long as the density ratio of negative ion to electron exceeds 50. For similar electronegativities the etching rates by negative ions is very close to that by positive ions. These results suggest that a low density plasma $\left(10^{14}-10^{16} \mathrm{~m}^{-3}\right)$ is transiting from a distinct behaviour associated to discharges with low and medium values for electronegativity to a negative ion dominated regime (ion-ion 
Highly electronegative plasmas

plasmas) when the electron fraction is less than $2 \%$ of the negative ion density. This value for electronegativity can be regarded as a requested parameter for applications that involves negative sheaths and negative ion etching at heat fluxes comparable to those by positive ions.

\section{Acknowledgments}

This work was partially supported by the Marie Curie International Reintegration Grant, project No. 46409.

\section{References}

[1] Economou D J 2007 Appl. Surf. Sci. 2536672

[2] Kono A 2002 Appl Surf. Sci. 192115

[3] Stamate E and Sugai H 2005 Phys. Rev. E 72036407

[4] Stamate E and Sugai H 2005 Phys. Rev. Lett. 94125004

[5] Stamate E, Ion beam extraction by discrete ion focusing, patent pending 09153323.2

[6] Stamate E 2009 Bull. American Phys. Soc. 5431

[7] Woolsey G A, Plumb I C, and Lewis D B 1973 J. Phys. D: Appl. Phys. 61883

[8] Kanakasabapathy S K, Overzet L J, Midha V, Economou D 2001 Appl. Phys. Lett. 7822

[9] Oohara W and Hatakeyama R 2003 Phys. Rev. Lett. 91205005

[10] Chabert P 2007 WO2007065915

[11] Leray G, Chabert P, Lichtenberg A, Lieberman M 2009 Bull. American Phys. Soc. 5442

[12] Charles C 2009 J. Phys. D: Appl. Phys. 42163001

[13] Keidar M, Raitses Y, Gallimore A D, Boeuf J P 2008 IEEE Trans.Plasma Sci. 361962

[14] Vozniy O V and Yeom G Y 2009 Appl. Phys. Lett. 94231502

[15] Dorf L, Raitses Y and Fisch N J 2005 J. Appl. Phys. 97103309

[16] Conde L, Fontán C F, Lambás J 2006 Phys. Plasmas 13113504

[17] Charles C 2007 Plasma Sources Sci. Technol. 16 R1

[18] Baalrud S D, Longmier B and Hershkowitz N 2009 Plasma Sources Sci. Technol. 18035002

[19] Pomot C, Mahi B, Petit B, Arnal Y, and Pelletier J 1986 J. Vac. Sci. Technol. B 41

[20] Pinto R, Ramanathan K V, and Babu R S 1987 J. Electrochem. Soc. 134165

[21] Scofield J D, Bletzinger P, and Ganguly B N 1998 Appl. Phys. Lett. 7376

[22] Khan F A and Adesida I 1999 Appl. Phys. Lett. 752268

[23] Khan F A, Roof B, Zhou L, and Adesida I 2001 J. Electronic Mat. 30212

[24] Chabert P 2001 J. Vac. Sci. Technol. B 191339

[25] Hsiao R, Miller D, Nguyen S, and Kellock A 1999 Appl. Surf. Sci. 1481 
Highly electronegative plasmas

[26] Shindo H, Sawa Y, and Horiike Y 1995 Jpn. J. Appl. Phys. 34 L925

[27] Ahny T H, Nakamura K, and Sugai H 1996 Plasma Sources Sci. Technol. 5139

[28] Samukawa S and Mieno T 1996 Plasma Sources Sci. Technol. 5132

[29] Kanakasabapathy S K, Khater M H, Overzet L J 2001 Appl. Phys. Lett. 791769

[30] Abe H, Yoneda M, and Fujiwara N 2008 Jap. J. Appl. Phys. 471435

[31] Gogolides E and Sawin H H 1992 J. Appl. Phys. 723971 Gogolides E and Sawin H H 1992 J. Appl. Phys. 723988

[32] Kanakasabapathy S K and Overzet L J 1998 Plasma Sources Sci. Technol. 7289

[33] Takeuchi T, Iizuka S, and Sato N 1998 Phys. Rev. Lett. 8077

[34] Franklin R_N 2003 J. Phys. D: Appl. Phys. 362655

[35] Kaganovich I D, Ramamurthi B N, and Economou D J 2001 Phys. Rev. E 64036402

[36] Junck K L and Getty W D 1994 J. Vac. Sci. Technol. A 122767

[37] Bacal M 2000 Rev. Sci. Instrum. 713981

[38] Amemiya H 1990 J. Phys. D: Apl. Phys. 23999

[39] Stamate E and Ohe K 1998 J. Appl. Phys 842450

[40] Stamate E, Popa G and Ohe K 1999 Rev. Sci. Instr. 7058

[41] Amemiya H, Annaratone B M, Allen J E 1999 Plasma Sources Sci. Technol. 8179

[42] Crespo R M, Palop J I F, Hernandez M A, del Pino S B, Diaz-Cabrera J M, Ballesteros J 2006 J. Appl. Phys. 99053303

[43] Shindo M, Ichiki R, Yoshimura S, Kawai Y 2001 Thin Solid Films 390222

[44] Stamate E, Sugai H, Ohe K 2002 Appl. Phys. Lett. 803066

[45] Wong A Y, D’Angelo N, and Motley R W 1962 Phys. Rev. Lett. 9415

[46] Shindo M, Kawai Y 2002 Thin Solid Films 407204

[47] Hershkowitz N and Ghim (Kim) Y.-C 2009 Plasma Sources Sci. Technol. 18014018

[48] Noguchi M, Ariga K, Hirao T, Suanpoot P, Yamagata Y, Uchino K and Muraoka K 2002 Plasma Sources Sci. Technol. 1157

[49] Leung K N, Hershkowitz N, and MacKenzie K R 1976 Phys. Fluids 191045

[50] Hershkowitz N and Intrator T 1981 Rev. Sci. Instrum. 521629

[51] Leung K N, Ehlers K W, and Bacal M 1983 Rev. Sci. Instrum. 5456

[52] Stamate E and Ohe K 2001 Appl. Phys. Lett. 78153

[53] Stamate E, Ohe H 2002 J. Vac. Sci Technol. A 20661

[54] Stamate E, Inagaki K, Ohe K and Popa G 1999 J. Phys. D: Appl. Phys. 32671

[55] Kline L E, Davies D K, Chen C L, and Chantry P J 1979 J. Appl. Phys. 506789

[56] Chen F F, Chang J P 2003 Lecture Notes on Principles of Plasma Processing (New York, Kluwer Academic/Plenum Publishers) p 34

[57] Demidov V I, DeJoseph C A Jr, Simonov V Ya 2007 Appl. Phys. Lett. 91201503 
Highly electronegative plasmas

[58] Stangeby P C, Allen J E 1971 Nature Physical Science 23326

[59] Stamate E, Sugai H, Takai O, Ohe K 2004 J. Appl. Phys. 95830

[60] Strat M, Strat G and Gurlui S 1999 J. Phys. D: Appl. Phys. 3234 


\section{FIGURE CAPTIONS}

Figure 1. (a) Experimental setup: PM-permanent magnets; $\mathrm{C}_{1}, \mathrm{C}_{2}$-metallic cylinders; H-heater; PP-planar probe; LP1, LP2-Langmuir probes; I-insulators; (b) Detail of the magnetic filter.

Figure 2. (a) The ratio of the negative ion density to electrons for $150 \mathrm{~mA}$ and $500 \mathrm{~mA}$ in SP (continuous line) and TP (dashed line) for a constant Ar flow of $2 \mathrm{sccm}$; (b) the corresponding positive ion densities; (c) the corresponding electron temperatures.

Figure 3. Negative ion species as a function of the $\mathrm{SF}_{6}$ flow (a) $I_{\mathrm{d}}=150 \mathrm{~mA}$ and (b) $I_{\mathrm{d}}=500 \mathrm{~mA}$, for a constant Ar flow of $2 \mathrm{sccm}$.

Figure 4. (a) Plasma potential drift in time for different flow rates of $\mathrm{Ar} / \mathrm{SF}_{6}$ at constant ratio 8; (b) the electronegativity corresponding to the same flow rates of $\mathrm{Ar} / \mathrm{SF}_{6}$ from (a); (c) plasma potential drift after cleaning the reactor chamber. The discharge current is $150 \mathrm{~mA}$ for all curves.

Figure 5. Plasma potential relaxation after it was brought down to $-20 \mathrm{~V}$ for two different temperatures of the inner metallic cylinders.

Figure 6. (a) Plasma potential as a function of $U_{\mathrm{W}}$ for different $\mathrm{SF}_{6}$ flow rates; (b) corresponding ion densities. For all curves Ar flow is $2 \mathrm{sccm}, I_{\mathrm{d}}=150 \mathrm{~mA}$ and the values in parentheses correspond to $\square$

Figure 7. Radial profile of the plasma potential and electron temperature before $\left(U_{\mathrm{W}}=10 \mathrm{~V}\right)$ and after the jump $\left(U_{\mathrm{W}}=25 \mathrm{~V}\right)$ corresponding to curve $\mathrm{SF}_{6}=0$ from figure 6 , for $\mathrm{Ar}=2 \mathrm{sccm}$ and $I_{\mathrm{d}}=150 \mathrm{~mA}$.

Figure 8. Current-voltage characteristics recorded by planar probe for different discharge currents in Ar plasma for a constant flow of $2 \mathrm{sccm}\left(\mathrm{SF}_{6}=0 \mathrm{sccm}\right)$.

Figure 9. Current-voltage characteristics recorded by planar probe for a discharge current $I_{\mathrm{d}}=500 \mathrm{~mA}$ for different $\mathrm{SF}_{6}$ flow rates at a constant Ar flow of $2 \mathrm{sccm}$.

Figure 10. The plasma potential and $\square$ dependence on the $\mathrm{SF}_{6}$ flow for a discharge current of $500 \mathrm{~mA}$ for $V_{\mathrm{pp}}=0 \mathrm{~V}$ with filled squares and $V_{\mathrm{pp}}=200 \mathrm{~V}$ with filled circles.

Figure 11. Etching rates for positive and negative ions for discharge currents of 150 and $500 \mathrm{~mA}(\mathrm{Ar}=2$ $\left.\mathrm{sccm}, \mathrm{SF}_{6}=0.5 \mathrm{sccm}\right)$. 
Highly electronegative plasmas

Figure 12. AFM measurements for (a) positive ions and (b) negative ions after etching in $\mathrm{Ar} / \mathrm{SF}_{6}$ plasma ( $=206$ ) with $2 \mathrm{sccm}$ Ar flow, $0.5 \mathrm{sccm} \mathrm{SF} 6$ flow, $I_{\mathrm{d}}=500 \mathrm{~mA}$ and $50 \mathrm{eV}$ ion energy. 
M. Draghici and E. Stamate

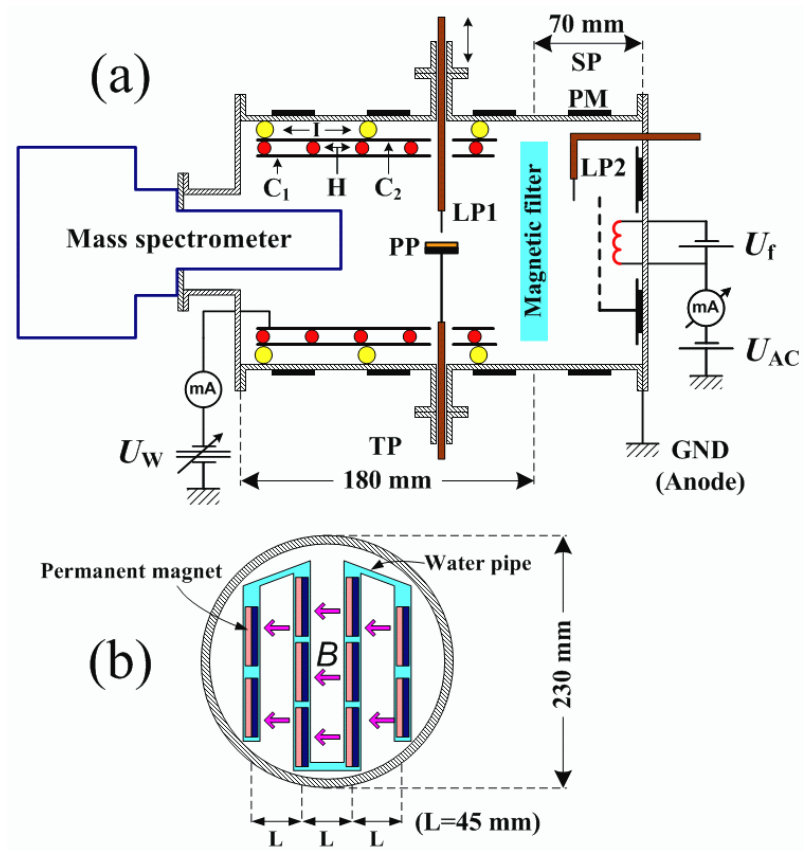

Figure 1. (a) Experimental setup: PM-permanent magnets; $\mathrm{C}_{1}, \mathrm{C}_{2}$-metallic cylinders; H-heater; PP-planar probe; LP1, LP2Langmuir probes; I-insulators; (b) Detail of the magnetic filter. 


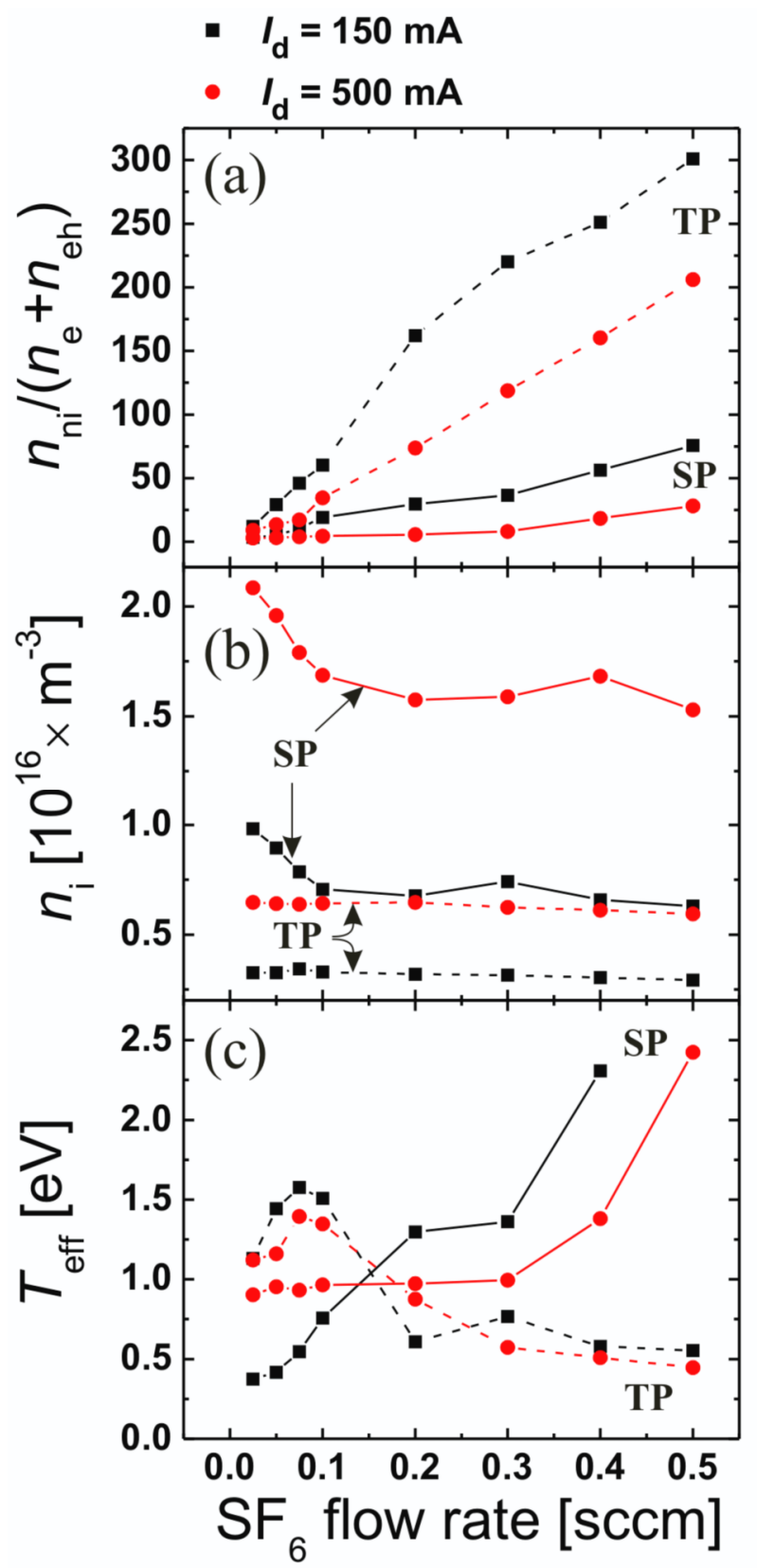

Figure 2. (a) The ratio of the negative ion density to electrons for $150 \mathrm{~mA}$ and $500 \mathrm{~mA}$ in SP (continuous line) and TP (dashed line) for a constant Ar flow of $2 \mathrm{sccm}$; (b) the corresponding positive ion densities; (c) the corresponding electron temperatures. 
M. Draghici and E. Stamate

(a)

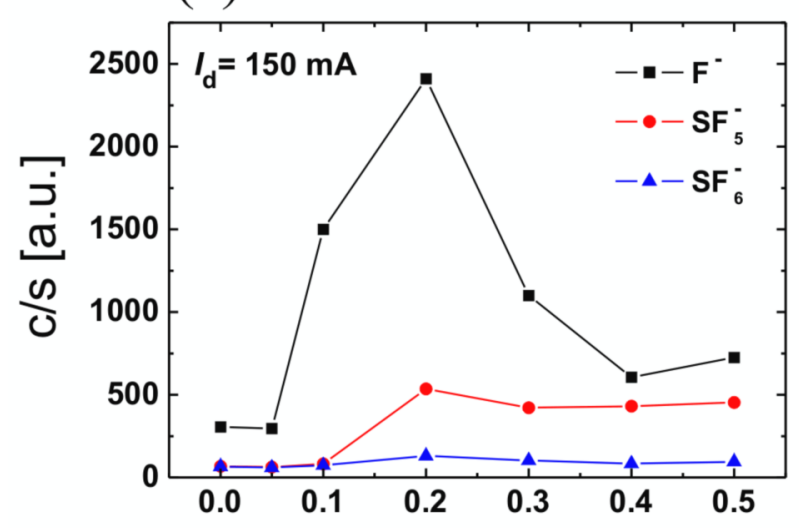

(b)

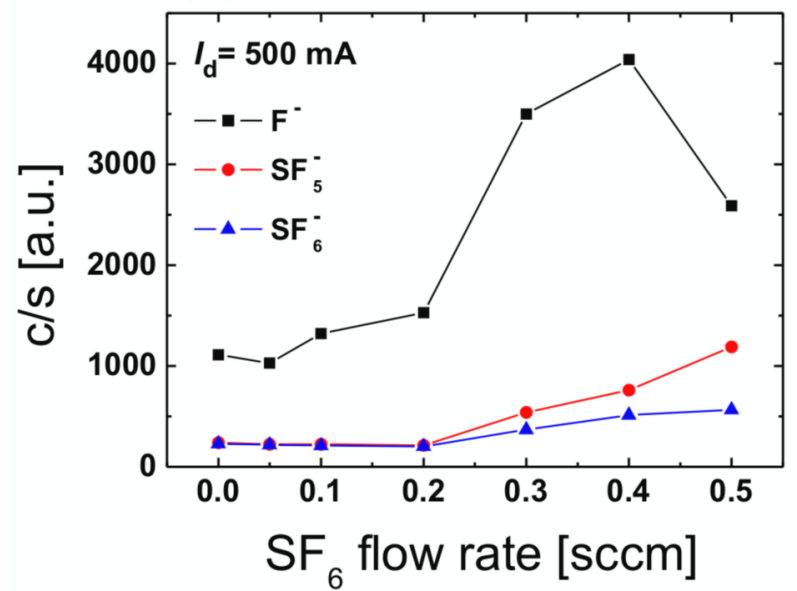

Figure 3. Negative ion species as a function of the $\mathrm{SF}_{6}$ flow (a) $I_{\mathrm{d}}=150 \mathrm{~mA}$ and (b) $I_{\mathrm{d}}=500 \mathrm{~mA}$, for a constant Ar flow of 2 sccm. 
M. Draghici and E. Stamate

(a)

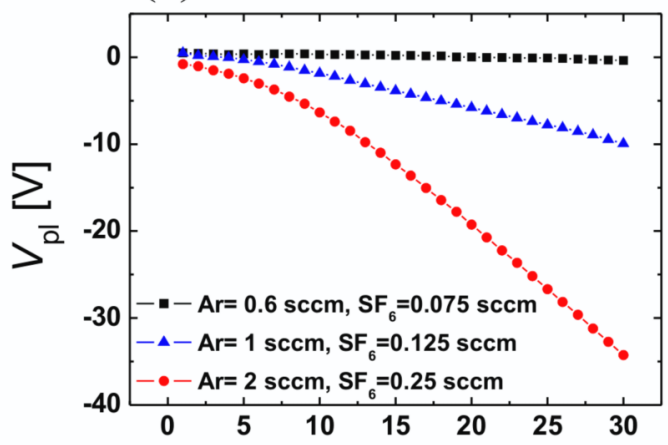

(b) Time [min]
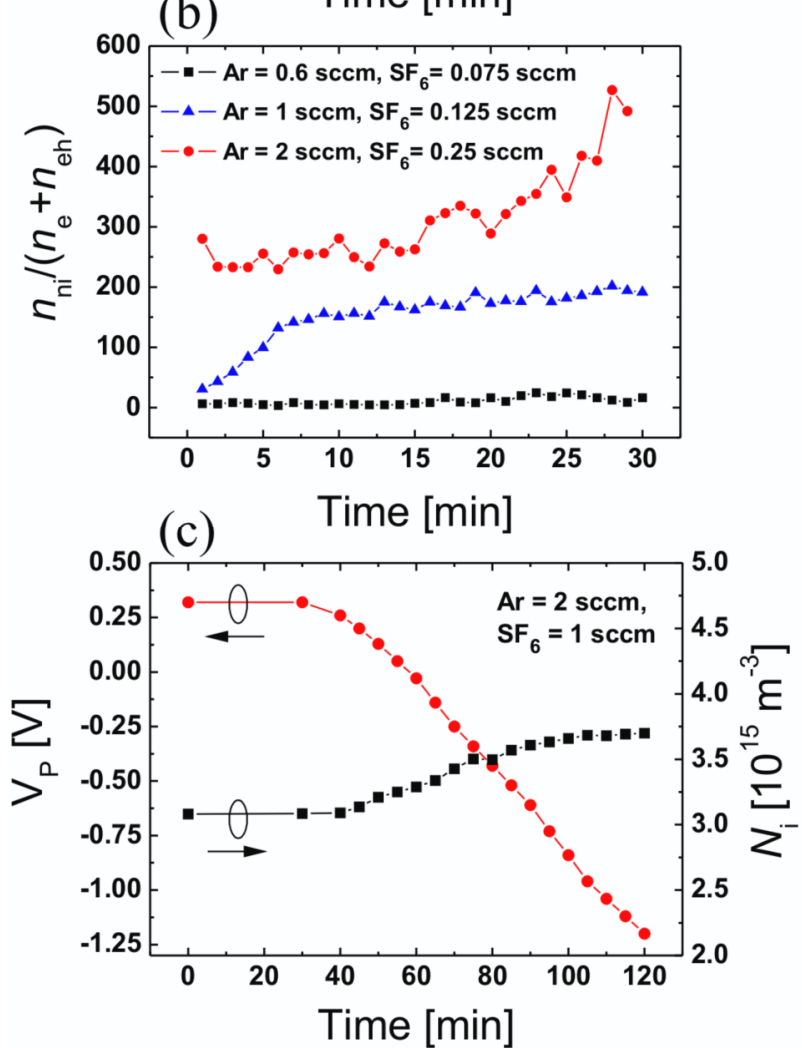

Figure 4. (a) Plasma potential drift in time for different flow rates of $\mathrm{Ar} / \mathrm{SF}_{6}$ at constant ratio 8; (b) the electronegativity corresponding to the same flow rates of $\mathrm{Ar} / \mathrm{SF}_{6}$ from (a); (c) plasma potential drift after cleaning the reactor chamber. The discharge current is $150 \mathrm{~mA}$ for all curves. 
M. Draghici and E. Stamate

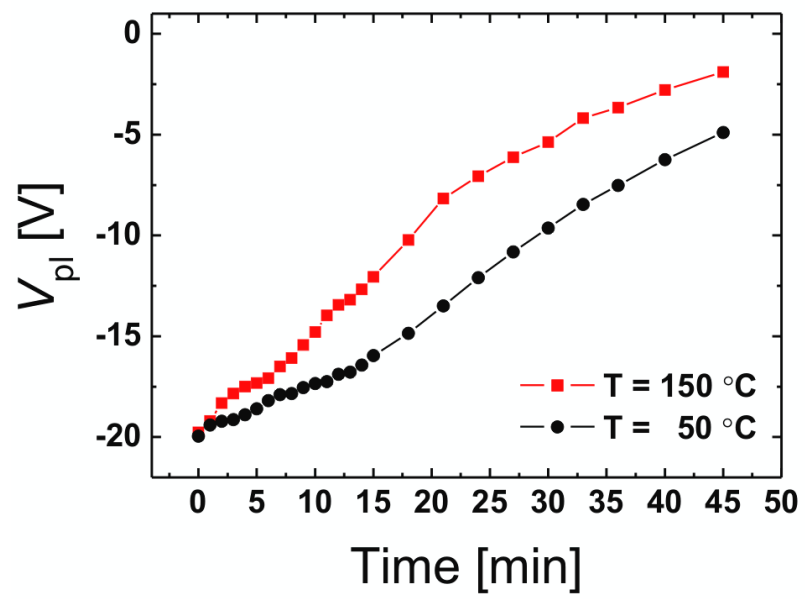

Figure 5. Plasma potential relaxation after it was brought down to $-20 \mathrm{~V}$ for two different temperatures of the inner metallic cylinders. 
M. Draghici and E. Stamate

(a)
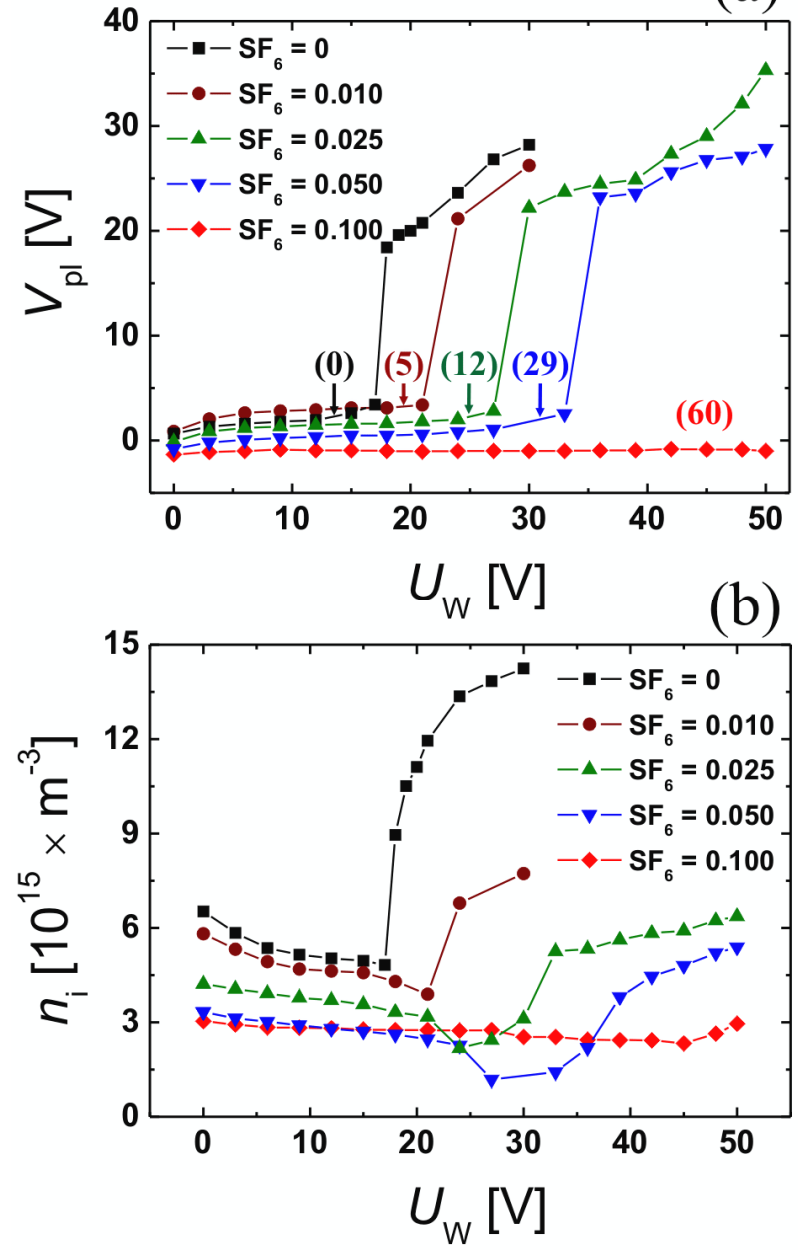

Figure 6. (a) Plasma potential as a function of $U_{\mathrm{W}}$ for different $\mathrm{SF}_{6}$ flow rates; (b) corresponding ion densities. For all curves Ar flow is $2 \mathrm{sccm}, I_{\mathrm{d}}=150 \mathrm{~mA}$ and the values in parentheses correspond to $\square$. 
M. Draghici and E. Stamate

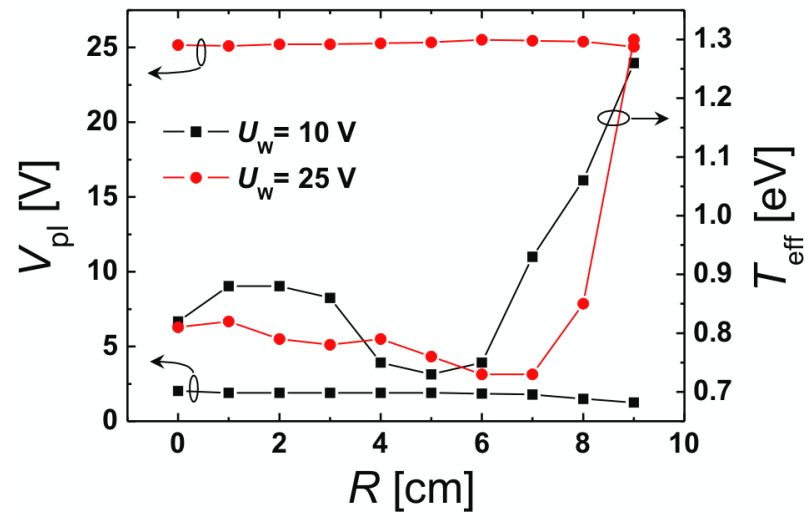

Figure 7. Radial profile of the plasma potential and electron temperature before $\left(U_{\mathrm{W}}=10 \mathrm{~V}\right)$ and after the jump $\left(U_{\mathrm{W}}=25 \mathrm{~V}\right)$ corresponding to curve $\mathrm{SF}_{6}=0$ from figure 6 , for $\mathrm{Ar}=2 \mathrm{sccm}$ and $I_{\mathrm{d}}=150 \mathrm{~mA}$. 
M. Draghici and E. Stamate

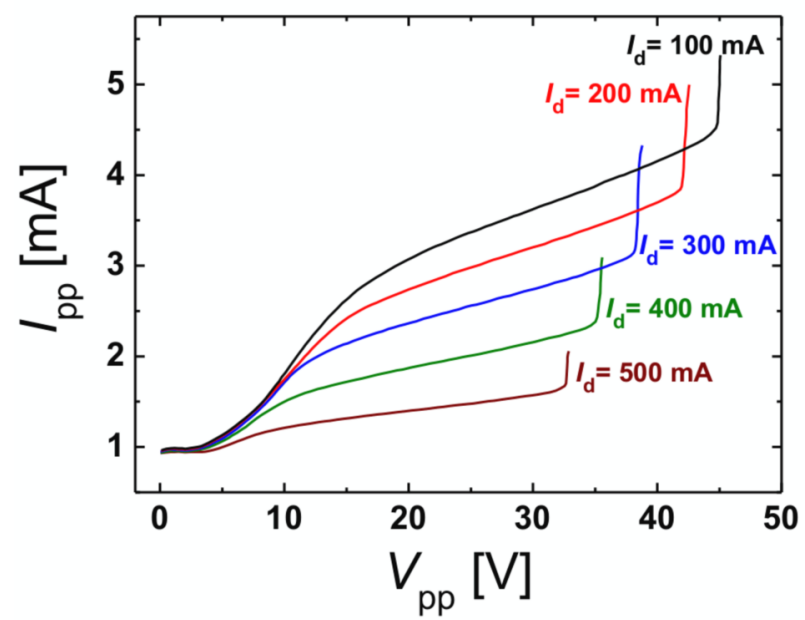

Figure 8. Current-voltage characteristics recorded by planar probe for different discharge currents in Ar plasma for a constant flow of $2 \mathrm{sccm}\left(\mathrm{SF}_{6}=0 \mathrm{sccm}\right)$. 
M. Draghici and E. Stamate

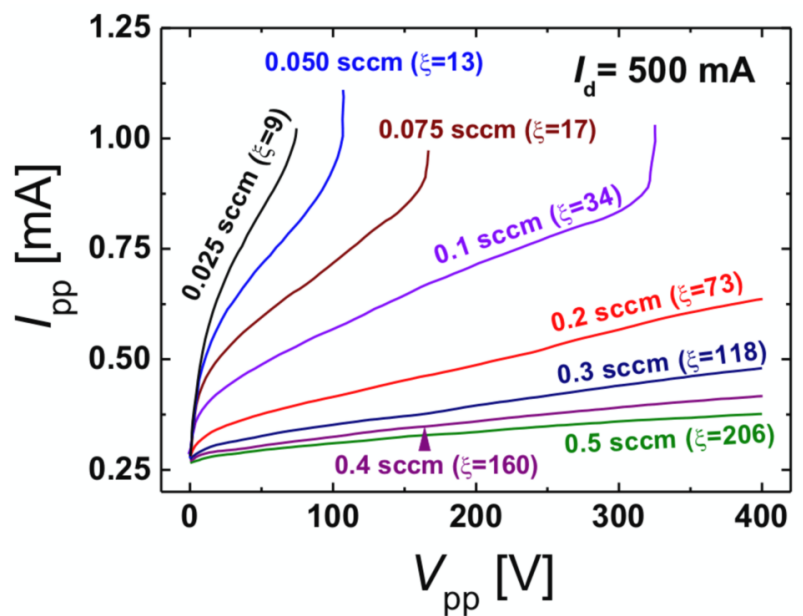

Figure 9. Current-voltage characteristics recorded by planar probe for a discharge current $I_{\mathrm{d}}=500 \mathrm{~mA}$ for different $\mathrm{SF}_{6}$ flow rates at a constant Ar flow of $2 \mathrm{sccm}$. 
M. Draghici and E. Stamate

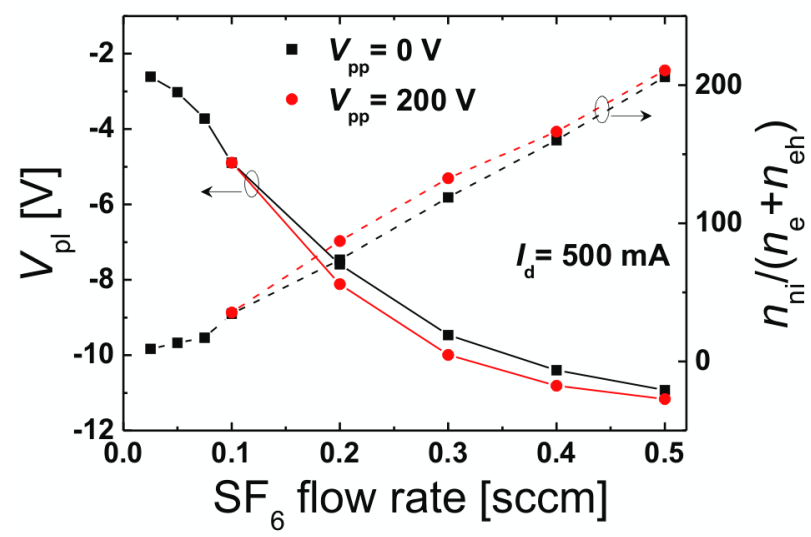

Figure 10. The plasma potential and $\square$ dependence on the $\mathrm{SF}_{6}$ flow for a discharge current of $500 \mathrm{~mA}$ for $V_{\mathrm{pp}}=0 \mathrm{~V}$ with filled squares and $V_{\mathrm{pp}}=200 \mathrm{~V}$ with filled circles. 
M. Draghici and E. Stamate

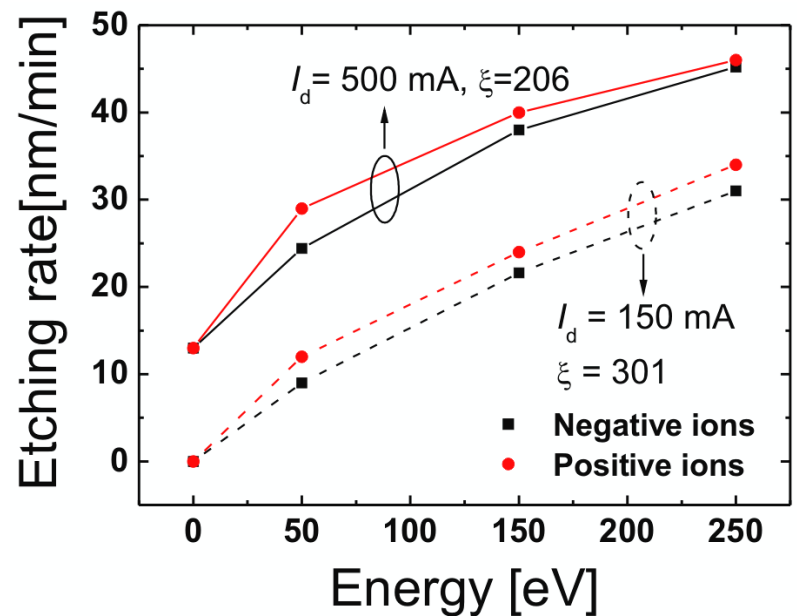

Figure 11. Etching rates for positive and negative ions for discharge currents of 150 and $500 \mathrm{~mA}\left(\mathrm{Ar}=2 \mathrm{sccm}, \mathrm{SF}_{6}=0.5\right.$ sccm). 
M. Draghici and E. Stamate

Topography - Scan forward

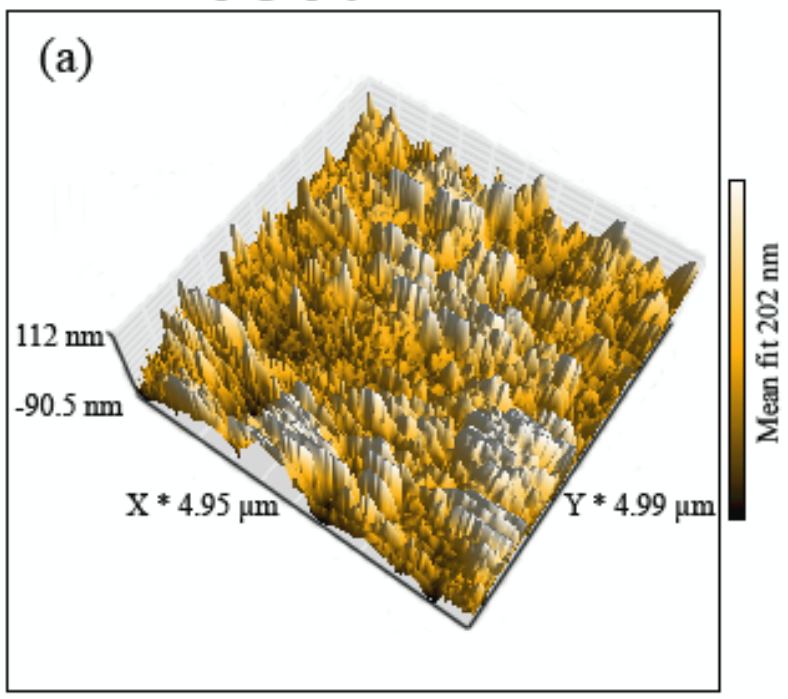

Topography - Scan forward

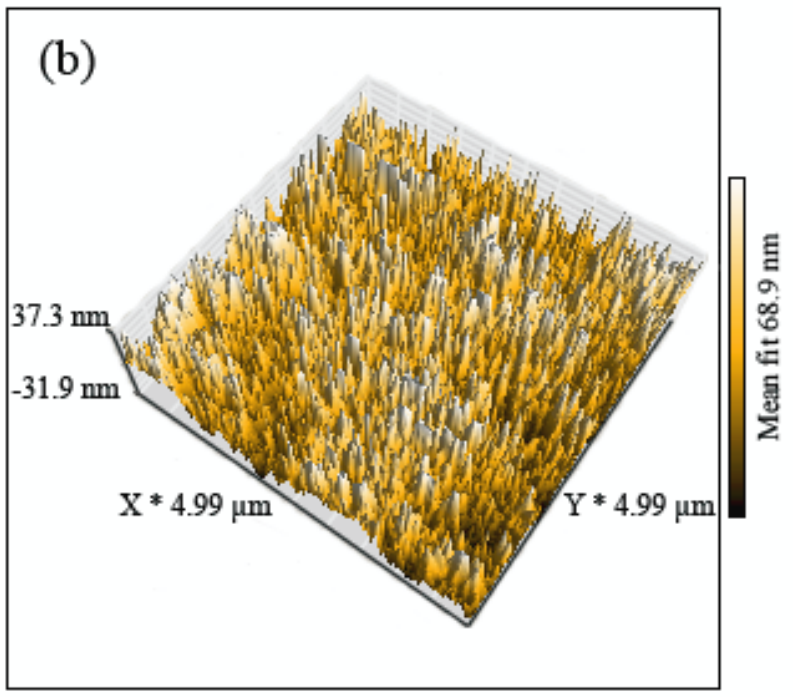

Figure 12. AFM measurements for (a) positive ions and (b) negative ions after etching in $\mathrm{Ar} / \mathrm{SF}_{6}$ plasma $(\not 206)$ with 2 sccm Ar flow, 0.5 sccm $\mathrm{SF}_{6}$ flow, $I_{\mathrm{d}}=500 \mathrm{~mA}$ and $50 \mathrm{eV}$ ion energy. 